\title{
Technologies in the Built Environment to Enable Sustainability and Energy Conservation
}

\author{
Mahmoud Alahmad, \\ University of Nebraska- \\ Lincoln \\ Hamid Sharif \\ University of Nebraska- \\ Lincoln
}

\author{
Timothy Wisnieski, \\ University of Nebraska- \\ Lincoln
}

\author{
Nasser Aljuhaishi \\ Kuwait University \\ Jonathan Shi, \\ University of Florida
Jill Neal
US Environmental Protection
Agency

\begin{abstract}
Fundamental understandings of how energy is consumed, monitored, and controlled are key prerequisites for an energy conservation process in the built environment. This paper discusses the use of real-time energy monitors (RTM) to influence behavior change in residential consumers. Furthermore, a bottom-up approach to real-time energy monitoring by integrating virtual and physical domains to increase user awareness on where, when, how and why aspect of energy to make inform decisions regarding energy consumption, optimization and conservation is proposed. A virtual 3-D environment is developed to display actual space/zone/building real-time power consumption information and to allow users to easily locate equipment/loads that are in standby/inefficient and causing energy waste in the real/physical environment. The proposed system using wireless ZigBee based monitoring system is demonstrated via a prototype board virtually integrated with a real world test environment. The results establish a promising tool in this filed.
\end{abstract}

\section{Introduction}

According to United States Green Building Council (USGBC), the built environment in the United States accounts for $72 \%$ of electricity consumption, $39 \%$ of energy use, $38 \%$ of all CO2 emissions, $40 \%$ of raw materials use, $30 \%$ of waste output (136 million tons annually), and $14 \%$ of potable water consumption [1]. U.S. homes use about one-fifth of the total energy consumed in the nation and about $60 \%$ of that is in the form of electricity. Research has shown that these residential energy consumers waste almost $41 \%$ of the power supplied to their homes [2]. The large amount of usage and waste indicates that the residential sector has significant energy savings potential. To this end, the development of smart buildings or home automation technologies promises to make our living environment safer, more convenient, comfortable and more efficient. Some of the most important functions of smart buildings include environment monitoring, building security, monitoring and control of power systems etc. In addition to these, reducing energy consumption in buildings offers a ready-to-use solution for reducing the effects of climate change and the potential of natural disasters and hazards.

Data communication systems and information technologies are essential components of modern smart buildings. The use of occupancy sensors and building automation sensors are two ways of using data communication systems to create a smart built environment. Occupancy sensors are simple devices that sense whether an occupant is in a space or not and transmit a signal wirelessly to energize or de-energize the equipment being controlled by these sensors, such as lights or ventilation systems. Building automation systems use the information from the sensors to provide significant reduction in energy usage and cost by offering automatic control of indoor environment conditions.

To influence the electrical energy consumption, energy efficiency programs in the U.S. emphasize the use of energy saving technologies and design practices because energy saved is, quite literally, energy found. A reduction in energy consumption will occur as a 
result of changing old habits as it is essential to influence human behavior since when it comes to energy, it is the habitual behavior that influences our energy consumption. This need has opened ample opportunities for research on creative methods that encourage more energy efficient lifestyles. In addition, the use of modern building automation systems has become essential in order to provide occupancy comfort while improving energy and equipment efficiency as well as reduce operation costs.

In the residential sector, research is focusing on monitoring, data collection and control. In [3], a model for residential energy consumption is discussed. The discussion focuses on major end-uses of secondary energy and identifies space heating, domestic hot water (DHW) and appliance and lighting (AL) loads. A hybrid modeling approach based on Neural Networks is developed to estimate DHW and AL national consumption for Canadian houses. In [4], a time-correlated appliance use is used to develop a domestic electricity demand model. In [5], a regression model of electricity consumption of housing units is developed.

Home automation and smart home research is advancing energy monitoring and control systems as a way to enable consumers to have more control over the amount of energy they consume. The authors of [6] introduce “QUEEN”, a real-time power monitoring tool for designing sustainable buildings that perform efficiently through optimization of the HVAC system. Another study proposes the Cluster Energy Management System (CEMS), a real-time power monitoring system able to optimize the energy usage of a zone by maintaining a balance between the supply and demand sides. The system is unique in that it links several home energy management systems (HEMS) together. CEMS is created using the Keio University Network Oriented Intelligent and Versatile Energy Saving System (KNIVES), a demand side management system that controls electronic devices based on co-operative and distributed control algorithms and real-time measurement variables [7]. In [8], the authors integrate the KNIVES system with a smart circuit box to predict residential power needs. Some emerging technologies at the outlet level [9-11], employ hardware additions to existing circuitry to achieve monitoring.

This paper addresses this behavior change to conserve energy and provide a case study conducted to determine the effect of real-time monitors to influence behavior change and educate homeowners on where energy is being used and how Information
Technologies can save wasted energy. A Real Time Power Monitoring system is proposed to enable the user to be empowered to make conscious decisions to make green, sustainable choices.

The paper is outlined as follows: the effectiveness of real-time energy monitors (RTM) is discussed in Section 2; Section 3 describes load profile forecasting as part of a non-intrusive data acquisition system. A Real Time Power Monitoring system is described in Section 4 along with a demonstrated prototype of the proposed system. Section 5 discusses areas for further research. Section 6 concludes the paper by discussions and remarks.

\section{Behavior change and energy consumption}

When it comes to human consumption behavior, information is power. In the area of feedback systems, several real-time monitors in the market present an opportunity to decrease energy consumption [12-17]. The use of real-time feedback presents an opportunity to decrease residential energy consumption by $10 \%$ $20 \%$ [18]. As can be seen from Fig. 1, lighting, heating, and cooling take up 58 percent of the annual energy bill for a typical household. The question posed by the Figure is an interesting question that current RTM are trying to address [19]. However, current RTM devices available in the market monitor and display overall energy consumption for the entire home only and do not have the capability to adaptively determine wasted energy consumption on behalf of the resident. Users are thus responsible for both determining the location of wasted energy and taking the necessary actions to reduce that waste.

A case study to investigate the selection and effect of RTM's on household electricity consumption behavior, promote energy conservation, assist consumers in reducing their energy usage and determine if real-time feedback will aid residential customers in reducing their energy usage is conducted in Omaha, Nebraska, U.S.A. from January 2008 to June 2010 [20]. It was hypothesized that consumers interested in reducing costs might respond to information about their energy consumption by engaging in energy saving behaviors during periods of higher demand. 
Annual Energy Bill for a typical Single Family Home is approximately $\$ 2,200$.
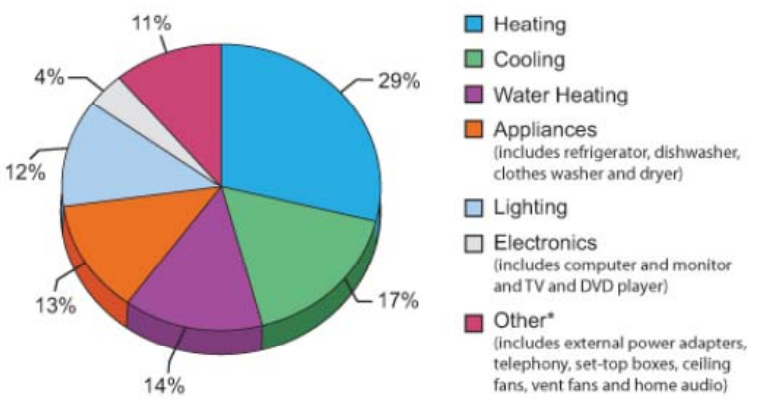

Fig.1.Energy consumption of a typical home [19]

The Aztech In-Home Display [13] and the PCM [14] devices were selected. During the study and based on the collected data, participants were grouped as low, medium, or high energy consumers. Figure 2 and Figure 3 show the consumption data collected on July 25, 2008, a typical summer day in Omaha, NE [21]. Peak usage occurred at different times in the day for different participants using these feedback devices. The study gave the real time energy consumption in 15 minute intervals. Beside the collected data, additional feedback from the participants was provided via two surveys conducted during the course of and at the end of the study. The survey looked at whether the real time monitors had any influence on participants desire to conserve energy. The results supported other studies that found that feedback needs to be more than just data presentation and needs to be more specific to appliance usage and that, as found in [22], that more innovative ways to reduce residential energy consumption are needed.

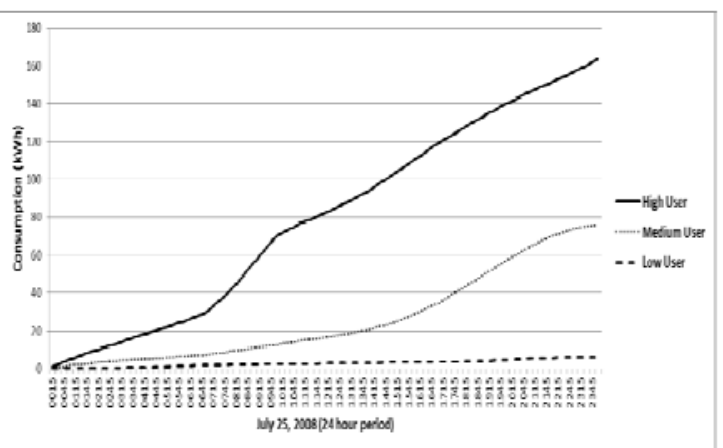

Fig. 2. Cumulative kWh for participants with Aztech monitors.

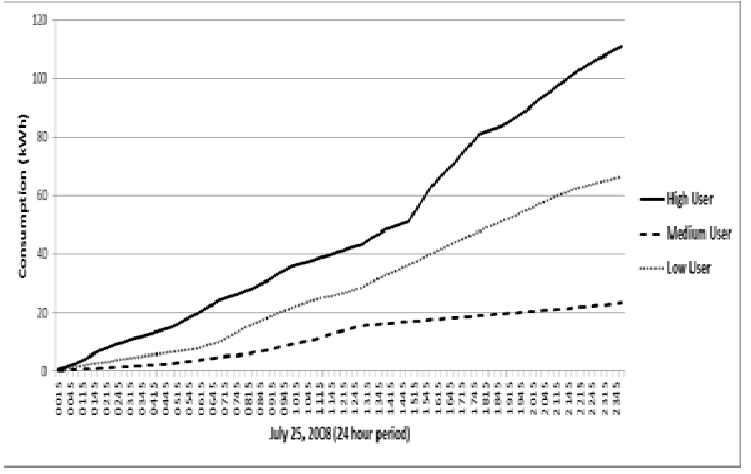

Fig. 3. Cumulative kWh for participants with PCM monitors.

\section{Load profile forecasting using ANN}

In the past, different application-specific methods have been investigated to understand, among other things, how loads affect power consumption, power quality, efficiency, reactive power compensation, and system planning. To gain an understanding and use this data in a real time comprehensive control system, load prediction and forecasting is needed. The purpose of the load prediction aspect of this research is to understand the characteristic-load consumption behavior. This means predicting the changes in the energy usage over time with respect to specific parameters. Now this window for the predicted energy usage will allow for the most suitable control strategies.

Electric load forecasting is the procedure used to forecast future loads, given past load data and outdoor weather information. Conventional methods used for load forecasting are designed based on relationships between load power and factors such as; occupancy, time of day and weather etc. These methods are able to forecast load power with modest accuracy using simple prediction models. However, since load power and the many influencing factors have a nonlinear relationship, it is difficult to achieve high accuracy and identify these non-linarites by using conventional methods.

Load forecasting methods [23, 24] based on similarity forecast future power load curve by using data of the day being matched to weather condition of the targeted day or forecast day. These kind of methods have the benefit of dealing with not only the nonlinear part of load power, but also with other factors like weekend and special days, outdoor air temperature, relative humidity etc. Within such prediction methods based on similarity, the power load 
on several selected similar days is averaged to increase the accuracy of load forecasting. Still, if there is a considerable error between similar day and the forecast day, the load power prediction method by means of averaging the load power on similar days will result in in-accurate measurements. Advanced approaches that perform better have been studied using neural networks for load forecasting [25].

Artificial Neural network are appropriate for load forecasting because of their approximation ability for nonlinear mapping and generalization. A neural network method will be employed to predict electrical loads for the built environment. The neural network will provide a $\mathrm{kW}$ Model. This model employs past trained data and serves as a baseline using past trained data to reflect an increase or decrease in energy use. The real-time monitored $\mathrm{kW}$ could be compared to the model kW (baseline). The difference between the two should reflect the potential energy savings. In other words, the Model kW will make it easier for engineers to define acceptable performance thresholds. Engineers could determine the operating schedule of electrical loads in the target system from measurements made at a centralized location.

The inputs for the training model are the past year reading of the building under study. For this simulation a building in Seattle, Washington, U.S.A. is chosen and used the daily temperature and humidity. The energy demand (in kilowatts) was sampled and stored every fifteen minutes. The weather parameters were provided by the local weather channel. Readings were collected every minute. The outside air temperature (OAT) and outside air relative humidity (ORAH) are two parameters employed in training our model. Hours and holidays were included as well as input parameters. Normalization was applied for all of the previous parameters the inputs were fed into our Artificial Neural Network (ANN) and after sufficient training were used to predict the load demand for the next year.

The target for this model was the demand $\mathrm{kW}$ and how that is correlated to the previous inputs. Figure 4 shows a block diagram representing the load forecasting process. Table 1 show the structure of the obtained Neural Network.

Figure 5 shows a plot of both the predicted and actual load in $\mathrm{kW}$ values against the hour of the day. The absolute mean error AME (\%) between the "predicted" and "Actual” loads for the predicted period has been calculated and presented in Table 1. Overall, the error values translate to an absolute mean error of $2.8 \%$ for the network. This represents a high degree of accuracy in the ability of neural networks to forecast electric load. The simulation results indicate that it's possible to implement this model in real time manner process. This will give instantaneous feedback for a potential control system. This in turn will reduce cost of upgrading and increase energy efficiency in the built environment. In future studies, it is expected that the proposed methods will lower energy spending by $15-25 \%$ spanning the lifecycle of most home electronics. This can be accomplished using only minimal additional hardware and software.

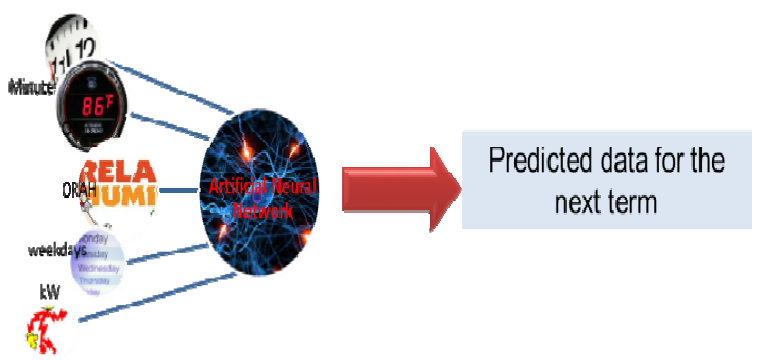

Fig. 4. General overview for the load forecasting model using ANN

Table 1 MLP structure

\begin{tabular}{cc}
\hline MLP STRUCTURE & \\
\hline Neural Network & MLP \\
Number of hidden layer & 1 \\
Number of hidden neuron & 17 \\
Activation function used in hidden layer & tan-sigmoid \\
Activation function used in output & lay \\
\hline
\end{tabular}

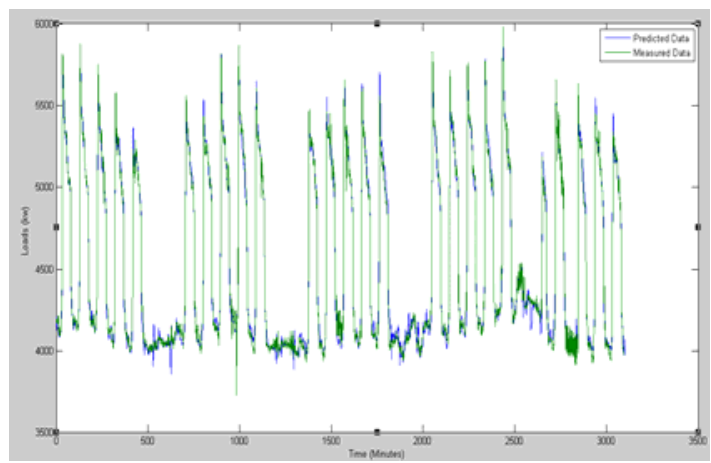

Fig. 5. Deviation of Predicted and Measured Energy data 


\section{Interactive real-time monitoring and control}

Much previous research has been devoted to improving ways to inform residents of their energy usage. To alleviate the information burden and guesswork about energy waste, an interactive real-time monitor and controller (RTMC) system as shown in Fig. 6 is proposed. This system achieves real-time energy monitoring by integrating virtual and physical domains to increase user's knowledge to make informed decisions regarding energy consumption, optimization and conservation. Using an innovative design and integration of physical and virtual domains, users will have easy access to the amount of energy they consume and the exact locale of this consumption. The system is demonstrated via a prototype board coupled with a virtual system. This energy monitoring system is based on an instantaneous impulse savings behavior that provides users with the ability to reduce energy usage in real time for the entire home by pressing a button. The system's user friendly interface with Go-green save-energy button is based on an adaptive system that monitors and controls real energy consumption at every energy consumption location or node. A node is defined as a point on the wiring system in the building at which electrical current is taken to supply utilization equipment (loads) [26]. Common nodes include receptacle, lighting switch, dedicated load, etc. This energy monitoring system will effectively determine non-critical and wasted energy in home and provide users with the ability to turn off wasted energy with the Go-green save-energy button. This will result in total power consumption for the home to be at its optimal level while also maintaining occupant comfort.

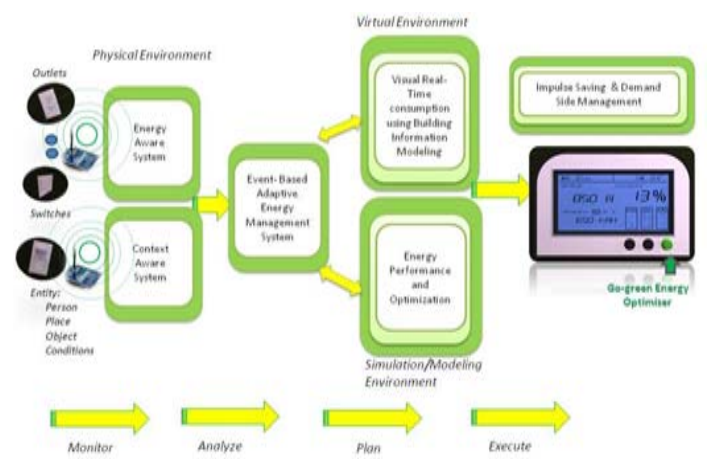

Fig. 6. Future vision for energy monitoring and control systems in smart homes.
The physical system consists of wireless nodes controlled by a central hub. Wireless nodes will transmit power consumption data from energy consuming devices to the central hub. This central hub will process all the information and bring the virtual and physical systems together. The hub will determine which devices could be turned off to save energy with data received by either a combination of occupancy sensors, temperature sensors, and lighting sensors, or by input from the user's personal preferences. By pressing the Go Green button the user can turn off all of the unneeded devices and save energy without the need of unplugging them manually. The hub will display the power consumption data in a format the user can easily comprehend with KW usage and dollar usage of the devices.

Wireless Data transmission is being pursued using Xbee radios [27]. These radios use the Zigbee protocol's [28] for wireless communication. They are low power radios that can monitor the current and voltage and act as an actuation device for each node with the help of triacs. This will make the nodes low in power consumption to maximize energy saved for each node. The low power of the radios does decrease their effective range but this is overcome by their ability to 'hop' data transmissions between each other until the data reaches the main hub for processing. The actuation system will use triacs to change the state of nodes with data from the central hub. This system will be small enough to be retrofitted into existing nodes in the form of an outlet cover or built into new buildings in the form of a new outlet box that fits existing outlet box sizes.

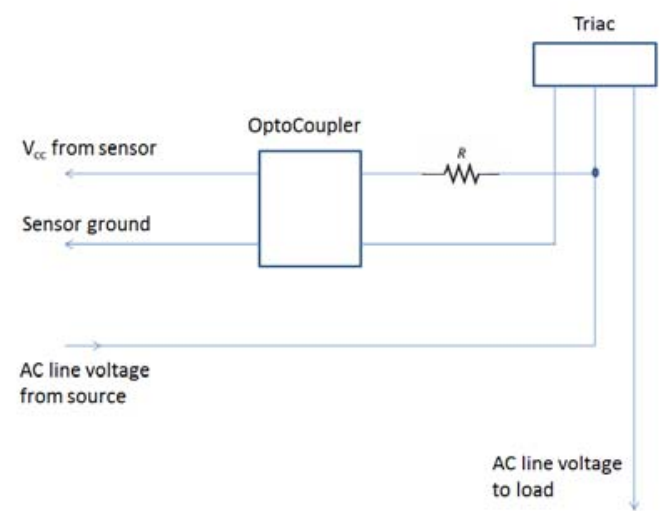

Fig. 7. AC control circuit 
Node actuation and control is accomplished using a triac circuit as shown in fig. 7. This circuit uses an optocoupler (OC) to interface with the traic and the AC power circuit. The OC is a device that allows the control and isolation of a signal by use of an internal LED activated transistor. The OC chosen has a built in zero-crossing detector which makes it excellent for use with triacs as they need to be activated at every zero crossing point along the AC cycle. The OC also acts as an isolation device between the DC sensor and the AC power signal. The advantages of this system over using a normal relay is that the control can be done with a simple low power signal instead of needing to use a high power signal to control the switching of the AC signal.

A simple wattmeter setup is used to measure the current and voltage of the AC signal. This gives a very accurate power reading to catch small loads that users may not know are even drawing power.

A prototype of this RTPMC system has been designed and tested. Fig. 8 shows the prototype board built to simulate six different loads. The prototype board also incorporates simulated occupancy, lighting, and temperature sensors in the room. These extra sensors help determine the optimum settings for the rooms loads.

The output of the board is integrated with a virtual environment model to illustrate the physical/virtual system. The virtual environment is built, using Visual Basic, for the prototype that emulates a room in a house. It provides the user with a simple interface that combines the actual layout of the room with real-time power consumption at every electrical node. Each load in this room is associated with one of the loads in the RTPMC prototype. When power is consumed at a load location, a red circle with a thickness proportional to the power consumption of that load will show up, indicating which load is consuming power in real time.

Fig. 9 shows real time power consumption by means of red circles, Excess (wasted) energy consumption is also highlighted at two locations. Although these two loads are turned off, they still consume power (phantom load). The red indicators function as warning sign for users to take action to conserve energy and minimize waste.

With the virtual environment the user has complete control over the loads in the building. Any node with a power sensor can have its state switched with the press of a button. Preset conditions from the user can be set and the users preferred optimum setting can be programmed-in so that automatic control of the room can be achieved without the user turning off each node individually.

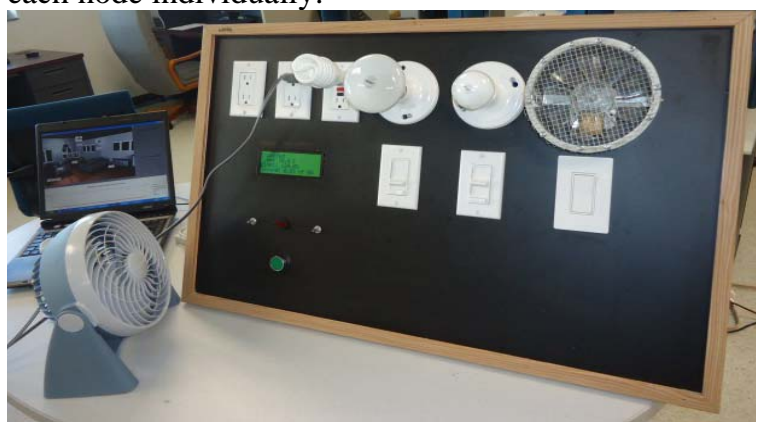

Fig. 8.The RTPM system prototype board.

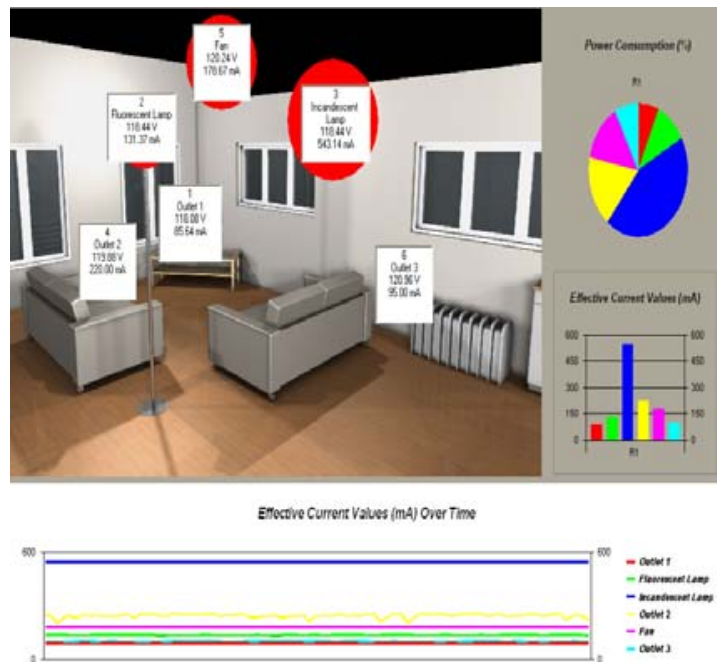

Fig. 9. The RTPMC physical/virtual real-time integration

This project distinguishes its approach by focusing on the user interface and experimentally verifying consumer behavior and achieving a significant energy saving. This will empower users to make their own decisions in order to simultaneously save money and energy using the RTPMC system. The RPTMC learns the occupant's energy behavior and determines the locations and magnitudes of wasted energy and provides this information in a user friendly approach to allow the user to take impulse action to sustain savings without having to estimate which action will best reduce energy use.

\section{Future work}

An event-based modeling and simulation of energy management systems is proposed to make the 
virtual model and the control logic a realistic resemblance of the actual system. This will provide a platform to foster research and perform experiments used to mimic the procedural manner with which a human brain perceives and reacts to real-life events. Humans use concern, satisfaction, and perception to make appropriate decisions to balance between energy consumption and conservation and human comfort. The perception and direct/indirect interaction of humans with the electrical system. The can be classified in an event-based model composed of:

- events, such as someone entering/leaving a room, a switch turning on/off, and a load being plugged into or out of an outlet;

- conditions/constraints, such as leaving lights on for security reasons, turning off the air conditioning (AC) if the temperature is less than a set point, and leaving on a computer that is in standby so as to prevent data loss;

- actions, such as changing the settings of the AC, increasing/decreasing fan speed, and shutting off phantom loads;

- states, such as on, off, speed 1, and speed 2 for a fan, or on, off, and dimmed for a light;

- data, such as the values of power consumption, voltage, currents, or power factor of the utilization equipment, the maximum power demand forecasted, the weather forecast, the electricity bill rates and tariffs and a fault diagnosed in some part of the electrical system; and

- transitions, such as a switch transitioning from state "on" to state "off," an occupancy sensor transiting from a state of working properly to a state of not working, and a motor transitioning from transient state to steady state from speed 1 to speed 2 , or from on to off state.

The main goal of this system is to minimize power consumption for the whole building. Power consumption can be expressed by Equation 1:

$$
P(n+1)=f(X(n), U(n))
$$

Where $\mathrm{P}(\mathrm{n}+1)$ is the total power consumption for the whole system at time step $n+1, X(n)$ is a matrix/array representing the current respective states of system's components at time step n, and U(n) is the system input matrix/array at time step $n$ (again including data, conditions and events).

\section{Conclusion}

In this paper, smart technologies are being introduced in the built environment in order to conserve energy and thus create a more sustainable future. Preliminary findings from the simulations indicate that a sensor technology can be developed successfully with the capacity to upgrade the existing electrical systems to provide real time monitoring. This technology will increase the energy efficiency in the built environment and eventually reducing greenhouse gas emissions $\mathrm{CO}_{2}, \mathrm{NO}_{2}$, etc. in the electrical power generation process.

\section{Acknowledgements}

This project is funded and sponsored by the United States Environmental Protection Agency (EPA) and University of Nebraska-Lincoln's Durham School of Architectural Engineerign and Cosntruction (DSAEC).

\section{References}

[1] U.S. Green Building Council (USGBC),"Green Building Facts." U.S. Green Building Council, "http://www.usgbc.org/ShowFile.aspx?DocumentID= 5961”, last accessed 2/3/2011.

[2] Williams, Eric D., \& Matthews, H. Scott., "Scoping the Potential of Monitoring and Control Technologies to Reduce Energy use in Homes," Proceedings of the 2007 IEEE International Symposium on Electronic \& Environment, May 2007, pp. 239-244.

[3] Lukas G. Swan, V. Ismet Ugursal, Ian BeausoleilMorrison, "Occupant related household energy consumption in Canada: Estimation using a bottom-up neural network technique," Journal of Energy and Buildings, Vol. 43, Issue 2-3, February-March 2011, pp. 326-337.

[4] Ian Richardson, Murray Thomson, David Infield, Conor Clifford, "Domestic Electricity Use: A highresolution energy demand model," Journal of Energy and Buildings, Vol.42, Issue 10, October 2010, pp. 1878-1887.

[5] Demba Ndiaye, Kamiel Gabriel, “ Principle component analysis of the electricity consumption in residential dwellings," Journal of Energy and 
Buildings, Vol. 43, Issue 2-3, February-March 2011, pp. 446-453.

[6] Markus Brychta, Florian Dubisch, Florian Stift, peter Palensky, "QUEEN - A Novel Design Flow and Decision Support Tool for Sustainable Buildings," Proceeding of the 36th Annual Conference of the IEEE Industrial Electronic Society (IECON 2010), Glendale, Arizona, 7-10 November, 2010.

[7] Yuto Kamiyoshi, Tomohisa Nakabe, Gouki Mine, Hiroaki Nishi, "Construction of Energy Measuring System in a University for Cluster Energy Management System," Distributed Demand Side Management System for Home Energy Management,” Proceeding of the 36th Annual Conference of the IEEE Industrial Electronic Society (IECON 2010), Glendale, Arizona, 7-10 November, 2010.

[8] Yukio Suhara, Tomohisa Nakabe, Gouki Mine, Hiroaki Nishi, "Distributed Demand Side Management System for Home Energy Management," Proceeding of the 36th Annual Conference of the IEEE Industrial Electronic Society (IECON 2010), Glendale, Arizona, 7-10 November, 2010.

[9]Intelli-plug, http://www.ethicalsuperstore.com/ products/oneclick/energy-saving-intelli-plug-dsk105/\#product_ethics, 2008.

[10] Sticord plugs, http://www.hometone.com/entry/ sticord-power-saver-plug-promises-to-conserveenergy/

[11] Vulcan Surge Protector, http://vulcantechnology.com/products/.

[12] Ambient Energy Orb, Ambientdevices, http://www.ambientdevices.com/cat/index.html

[13] Aztech Associates Incorporated, http://www.aztechinc.com/en/.

[14]Blueline Innovations, http://www.bluelineinnovations.com.

[15] Wattson device, http://www.diykyoto.com/uk.

[16]The Energy Detective, http://www.theenergydetective.com.

[17] Cent-A-Meter http://www.centameter.co.nz/.
[18]Wallace, James (2007). “TED the energy detective."http://www.currentcost.net/\%202818000.sh tml

[19] Energy Star 2009. "Where Does my Money Go.” http://www.energystar.gov/index.cfm?c=products.pr_p ie.

[20] M. Alahmad, Andrea Wilkerson, Josh Eiden, "Collaboration with Industry to Promote Energy Conservation and Education", Proceedings of the Annual American Society for Engineering Education (ASEE), Austin, Texas, June 14-17, 2009.

[21] Mahmoud Alahmad, Muhammad Zulfiqar, Hosen Hasna, Timothy Wisnieski, Nasser Aljuhaishi, Hamid Sharif, "Technologies Enabling Sustainability in The Built Environment," 6th International Conference for Internet Technology and Secured Transactions (ICITST-2011) Abu Dhabi, UAE, December 11-14, 2011.

[22] Karen Ehrhardt-Martinez, Kat A. Donnelly, \& John A. "Skip” Laitner, “Advanced metering Initiatives and Residential Feedback Programs: A Meta-Review of Household Electricity-Saving Opportunities," American Council for an EnergyEfficient Economy, report Number E105, June 2010.

[23]T. Senjyu, S. Higa, T. Yue-Jin, and K. Uezato, "Future load curve shaping based on similarity using Fuzzy logic approach,” in Proc. Int. Power Eng. Conf (IPEC), vol. II, 1997, pp. 483-488.

[24] S. Rahman and G. Shrestha, “A priory vector based technique for load forecasting," IEEE Trans. Power Syst., vol. 6, pp. 1459-1464, Nov. 1993.

[25]A. S. AlFuhaid, M. A. El-Sayed, and M. S. Mahmoud, "Cascaded artificial neural networks for short-term load forecasting,” IEEE Trans. Power Syst., vol. 12, pp. 1524-1529, Nov. 199

[26] Mark W. Earley, Jeffrey S. Sargent, Joseph V. Sheehan, E. William Buss, "National Electrical Code Handbook,” NEC 2008, NFPA, pp 29.

[27] Xbee Radios, http://www.digi.com/products/ wireless-wired-embedded-solutions/zigbee-rfmodules/point-multipoint-rfmodules/xbee-series1module\#overview.

[28] Zigbee Protocol, http://www.zigbee.org/ 